\title{
The configuration space of a robotic arm in a tunnel of width 2
}

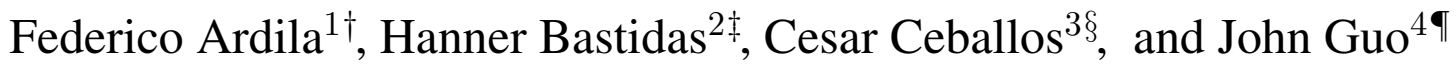

${ }^{1}$ Department of Mathematics, San Francisco State University, USA and Universidad de Los Andes, Colombia.

${ }^{2}$ Departamento de Matemáticas, Universidad del Valle, Colombia.

${ }^{3}$ Faculty of Mathematics, University of Vienna, Austria.

${ }^{4}$ Department of Mathematics, San Francisco State University, USA.

\begin{abstract}
We study the motion of a robotic arm inside a rectangular tunnel of width 2 . We prove that the configuration space $\mathcal{S}$ of all possible positions of the robot is a CAT(0) cubical complex. Before this work, very few families of robots were known to have $\mathrm{CAT}(0)$ configuration spaces. This property allows us to move the arm optimally from one position to another.

Ardila, Owen, and Sullivant gave a bijection between CAT(0) cubical complexes and posets with inconsistent pairs (PIPs), and we describe the coral PIP which corresponds to $\mathcal{S}$ under this bijection. We also compute the $f$-vector of $\mathcal{S}$ and use it to verify that the Euler characteristic of $\mathcal{S}$ equals 1.

Résumé. Nous étudions le mouvement d'un bras robotisé à 'intérieur d'un tunnel de largeur 2. Nous dmontrons que l'espace des configurations $\mathcal{S}$ consistant de toutes les positions du robot est un complexe cubique CAT(0). Très peu de familles de robots satisfont cette propriété; cette famille, précédemment hors d'atteinte, était la prochaine à être étudiée. Cette propriété nous permet the bouger le bras de façon optimale d'un position à l'autre.

Ardila, Owen et Sullivant ont donné une biection entre les complexes cubiques CAT(0) et les ensembles partiellement ordonns avec pair inconsistantes (PIPs) et nous décrivons les PIP coral qui correspond à $\mathcal{S}$ sous cette bijection. Nous calculons aussi le $f$-vecteur de $\mathcal{S}$ et l'utilisons pour vérifier que la caractéristique d'Euler de $\mathcal{S}$ est égale à 1.
\end{abstract}

Keywords. cubical complex, $\operatorname{CAT}(0)$, robot, poset with inconsistent pairs, $f$-vector

\footnotetext{
${ }^{\dagger}$ Email: federico@sfsu.edu. Partially supported by the US National Science Foundation CAREER Award DMS-0956178 and the SFSU-Colombia Combinatorics Initiative.

‡Email: hanner.bastidas@correounivalle.edu.co. This paper includes results from HB’s undergraduate thesis at U. del Valle, advised by FA and CC.

§Email: cesar.ceballoseunivie.ac.at. Partially supported by the government of Canada through a Banting Postdoctoral Fellowship, by a York University research grant, and by the Austrian Science Foundation FWF, grant F 5008-N15, in the framework of the Special Research Program "Algorithmic and Enumerative Combinatorics".

IEmail: jguo@mail.sfsu.edu. This paper includes results from JG's undergraduate applied math project at SFSU, advised by FA.
}

1365-8050 @ 2016 Discrete Mathematics and Theoretical Computer Science (DMTCS), Nancy, France 


\section{Introduction}

We consider a robotic arm $R_{n}$ of length $n$ moving in a rectangular tunnel of width 2 without selfintersecting. The robot consists of $n$ links of unit length, attached sequentially, and its base is affixed to the lower left corner. Figure 1 illustrates two possible positions of an arm of length 8 .
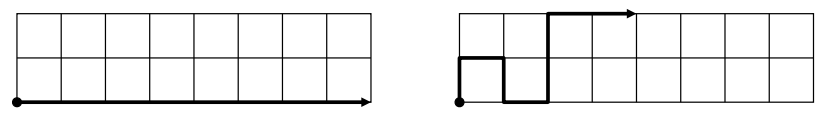

Fig. 1: Two possible positions of the robotic arm $R_{8}$.

The robot starts in the fully horizontal position, and is free to move using two kinds of local moves:

- Switching corners: Two consecutive links facing different directions interchange their directions.

- Flipping the end: The last link of the robot rotates $90^{\circ}$ without intersecting itself.
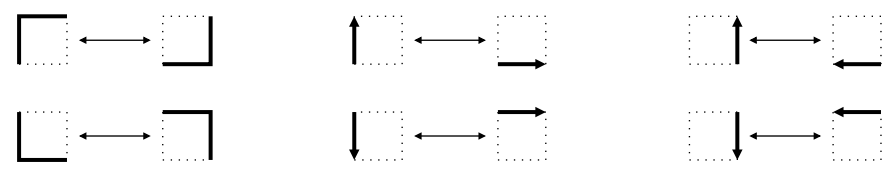

Fig. 2: The two kinds of local moves of the robotic arm.

We study the following fundamental problem.

Problem 1.1 Find the fastest way of moving the robotic arm $R_{n}$ from one position to another.

When we are in a city we do not know well and we are trying to get from one location to another, we will usually consult a map of the city to plan our route. This is a simple but powerful idea. Our strategy to approach Problem 1.1 will be to build and understand the "map" of all possible positions of the robot; this is called the configuration space or state complex $\mathcal{S}_{n}$. Following work of Reeves [9] and AbramsGhrist [1], Ardila, Baker, and Yatchak [2] showed that, if the configuration space of the robotic arm is CAT(0), Problem 1.1 can be solved efficiently. Thus Problem 1.1 motivates our main result.

Theorem 1.2 The configuration space $\mathcal{S}_{n}$ of the pinned-down robotic arm $R_{n}$ of length $n$ in a tunnel of width 2 is a $\mathrm{CAT}(0)$ cubical complex.

In Section 3 we collect some preliminary evidence for this theorem. It follows from very general results of Abrams and Ghrist [1] that the configuration space $\mathcal{S}_{n}$ is a cubical complex. Also, we know from work of Gromov [7] that $\mathcal{S}_{n}$ will be $\mathrm{CAT}(0)$ if and only if it is contractible. Therefore, before proving Theorem 1.2. we first verify that $\mathcal{S}_{n}$ has the correct Euler characteristic. We do it as follows.

Theorem 1.3 Let $\mathcal{S}_{n}$ be the configuration space for the robotic arm $R_{n}$ of length $n$ moving in a rectangular tunnel of width 2. If $c_{n, d}$ denotes the number of d-dimensional cubes in $\mathcal{S}_{n}$, then

$$
\sum_{n, d \geq 0} c_{n, d} x^{n} y^{d}=\frac{1+x^{2}+2 x^{3}-x^{4}+x y+x^{2} y+4 x^{3} y+x^{4} y+x^{3} y^{2}+2 x^{4} y^{2}+x^{5} y^{2}}{1-2 x+x^{2}-x^{3}-x^{4}-2 x^{4} y-2 x^{5} y-x^{5} y^{2}-x^{6} y^{2}} .
$$


Notice that the Euler characteristic of $\mathcal{S}_{n}$ is given by $\chi\left(\mathcal{S}_{n}\right)=c_{n, 0}-c_{n, 1}+\cdots$. Substituting $y=-1$ in the above formula, we obtain the generating function for $\chi\left(\mathcal{S}_{n}\right)$, which equals $1 /(1-x)=1+x+x^{2}+\cdots$. We conclude:

Corollary 1.4 The Euler characteristic of the configuration space $\mathcal{S}_{n}$ equals 1.

In Section 4 we sketch a proof of Theorem 1.2 Ardila, Owen, and Sullivant [4] gave a bijection between rooted CAT $(0)$ cubical complexes $X$ and simpler combinatorial objects $P(X)$ called posets with inconsistent pairs or PIPs. This bijection allows us to prove (rooted) cubical complexes are CAT $(0)$ by identifying their corresponding PIP. Following this approach, we show the following result which implies Theorem 1.2

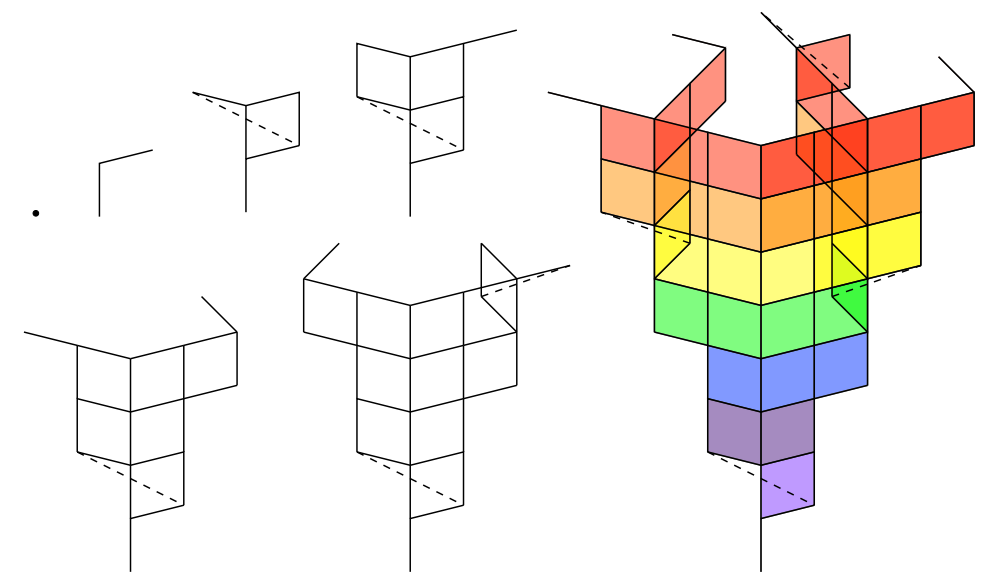

Fig. 3: The coral PIPs ("remote controls") for the robotic arms of length $1,2,3,4,5,6,9$ in a tunnel of width 2 .

Theorem 1.5 The PIP corresponding to the configuration space $\mathcal{S}_{n}$ for the robotic arm $R_{n}$ of length $n$ in a tunnel of width 2 is the extended coral PIP of Definition 4.4

We use the PIP $P(X)$ as a "remote control" to move the robot and navigate the space $X$. Using this remote control, we implement an algorithm to move the robotic arm in a tunnel of width 1 (using the results of [2]) and 2 (using Proposition 1.5] optimally, thus solving Problem 1.1

\section{Preliminaries: Configuration spaces and CAT(0) cube complexes.}

We begin by considering the transition graph $G\left(R_{n}\right)$ whose vertices are the possible states of the robot, and whose edges correspond to the allowable moves between them. Figure 4 and Figure 5 illustrate the transition graphs $G\left(R_{4}\right)$ and $G\left(R_{6}\right)$ of a robotic arm of length 4 and 6 respectively.

As these examples illustrate, each one of these graphs is the 1-skeleton of a cubical complex. For example, consider a position $u$ which has two legal moves $a$ and $b$ occuring in disjoint parts of the arm. We call $a$ and $b$ physically independent or commutative because $a(b(u))=b(a(u))$. In this case, there is a square connecting the vertices $u, a(u), b(a(u))=a(b(u))$, and $b(u)$ in $G\left(R_{n}\right)$. This is a general phenomenon, which we now make precise. 


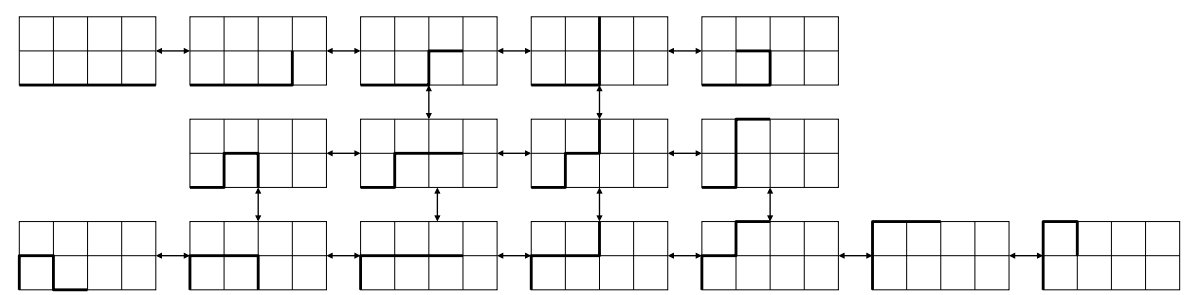

Fig. 4: The transition graph of the robotic arm of length 4 .

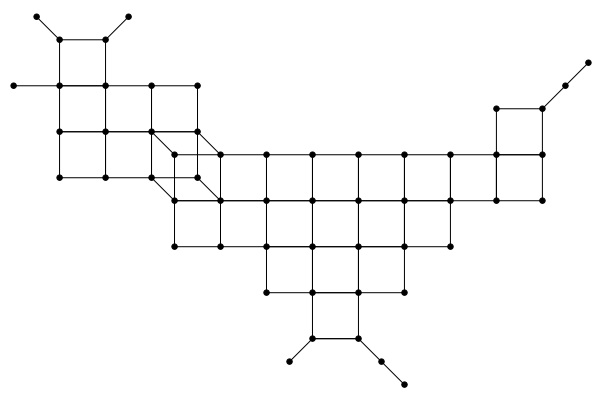

Fig. 5: The transition graph of the robotic arm of length 6 .

Definition 2.1 The configuration space or state complex $\mathcal{S}\left(R_{n}\right)$ of the robot $R_{n}$ is the following cubical complex. The vertices correspond to the states of $R_{n}$. An edge between vertices $u$ and $v$ corresponds to a legal move which takes the robot between positions $u$ and $v$. The $k$-cubes correspond to $k$-tuples of commutative moves: Given $k$ such moves which are applicable at a state $u$, we can obtain $2^{k}$ different states from u by performing a subset of these $k$ moves; these are the vertices of a $k$-cube in $\mathcal{S}\left(R_{n}\right)$.

Definition 2.2 A metric space $X$ is said to be CAT(0) if:

- there is a unique geodesic (shortest) path between any two points in $X$, and

- X has non-positive global curvature.

The second property, illustrated in Figure 6, may be described as follows. Let $T$ be a triangle in $X$ of side lengths $a, b, c$, and let $T^{\prime}$ be the triangle with the same lengths in the Euclidean plane. Consider a chord of length $d$ in $T$ which connects two points on the boundary of $T$; there is a corresponding comparison chord in $T^{\prime}$, say of length $d^{\prime}$. If $d \leq d^{\prime}$ for any chord in $T$, we say that $T$ is a thin triangle in $X$. The metric space $X$ has non-positive global curvature if every triangle in $X$ is thin.
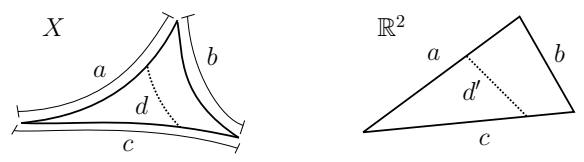

Fig. 6: A chord in a triangle in $X$, and the corresponding chord in the comparison triangle in $\mathbb{R}^{2}$. The triangle in $X$ is thin if $d \leq d^{\prime}$ for all such chords. 
The $\mathrm{CAT}(0)$ property is particularly favorable for configuration spaces, as the next result shows.

Theorem 2.3 [1] 2, 9] If the configuration space of a robot is a CAT(0) cubical complex, there is an algorithm to find the fastest way of moving the robot from one position to another.

As explained in [2], Theorem 2.3 applies to three natural definitions of "fastest", in terms of:

- the number of moves, if only one move at a time is allowed,

- the number of steps (where in each step we may perform several physically independent moves),

- time elapsed.

One could also ask for the shortest path between two points in a CAT(0) cubical complex under the Euclidean metric. This seems to be a more difficult problem, as explained by Ardila-Owen-Sullivant in [4]. In any case, the Euclidean metric is less relevant to robotic applications.

\section{Face enumeration and the Euler characteristic of $\mathcal{S}_{n}$}

Definition 2.2 does not provide an efficient way of testing whether a space is $\operatorname{CAT}(0)$. Gromov proved the groundbreaking result that for cubical complexes, this metric property has a topological-combinatorial characterization:

Theorem 3.1 [7] A cubical complex is $\mathrm{CAT}(0)$ if and only if it is simply connected, and the link of every vertex is a flag simplicial complex.

Recall that a simplicial complex $\Delta$ is flag if it has no empty simplices; that is, if the 1-skeleton of a simplex is in $\Delta$, then that simplex must be in $\Delta$. It is clear from the definition of $\mathcal{S}_{n}$ that the link of every vertex is flag. Furthermore, notice that non-simply connected spaces cannot be contractible, while $\mathrm{CAT}(0)$ spaces are contractible [5]. Therefore, Theorem 1.2 is equivalent to proving that $\mathcal{S}_{n}$ is contractible. In this section, we prove the following partial result in that direction.

Theorem 3.2 The Euler characteristic of the configuration space $\mathcal{S}_{n}$ equals 1.

\subsection{Face enumeration.}

Let us compute the generating function for the $f$-vectors of the configuration spaces $\mathcal{S}_{n}$.

\subsubsection{States of the robot with a link facing to the left.}

We first observe that the robotic arm may reach a state where the end of the arm is facing to the left as illustrated in Figure 7 However, since the robot never self intersects, we have at most one link facing left.

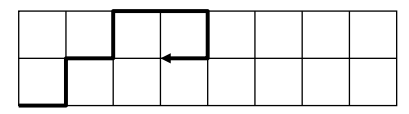

Fig. 7: A state of the robotic arm with a link facing to the left.

Lemma 3.3 The robotic arm always has at most one link facing to the left. If it does have one such link, the last three links of the arm must be in one of the two positions shown in Figure 8 


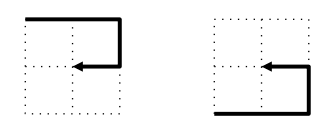

Fig. 8: Possible ends of a robotic arm with a link facing to the left.

\subsubsection{Cubes and partial states}

Consider a $d$-cube in the configuration space $\mathcal{S}_{n}$; it has $2^{d}$ vertices. If one superimposes the corresponding $2^{d}$ positions of the robotic arm, one obtains a sequence of edges, squares, and possibly a "claw" in the last position, as illustrated in Figure 9 The number of squares (including the claw if it is present) is $d$, corresponding to the $d$ physically independent moves that are being represented by this cube. We call the resulting diagram a partial state, and let its weight be $x^{n} y^{d}$. It is clear that the partial states of weight $x^{n} y^{d}$ are in bijection with the $d$-cubes of $\mathcal{S}_{n}$.

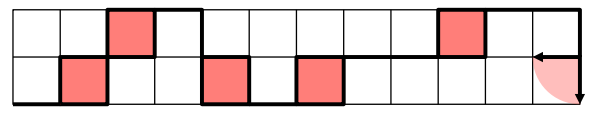

Fig. 9: A partial state corresponding to a 6 -cube in the configuration space $\mathcal{S}_{20}$.

Each partial state gives rise to a word in the alphabet $\{r, v, \ell, \square,\llcorner\lrcorner$,$\} , where:$

- $r$ represents a horizontal link of the robot facing to the right. Its weight is $x$.

- $v$ represents a vertical link. Its weight is $x$.

- $\ell$ represents a horizontal link facing to the left. Its weight is $x$.

- $\square$ represents a square, which comes from a move that switches corners of two consecutive links facing different directions. Its weight is $x^{2} y$.

- $\llcorner$ represents a claw, which comes from a move that flips the end of the robot, with the horizontal link facing to the right. Its weight is $x y$.

- $\lrcorner$ represents a claw, which comes from a move that flips the end of the robot, with the horizontal link facing to the left. Its weight is $x y$.

For example, the partial state of Figure 9 gives rise to the word $r \square \square r v \square r \square r r \square r r v\lrcorner$. The weight of the partial state is the product of the weights of the individual symbols; in this case it is $x^{20} y^{6}$, which is equal to the product $x\left(x^{2} y\right)\left(x^{2} y\right) x x\left(x^{2} y\right) x\left(x^{2} y\right) x x\left(x^{2} y\right) x x x(x y)$. It is worth remarking that this word does not determine the partial state uniquely.

\subsubsection{Factorization of partial states into irreducibles.}

Our next goal is to use generating functions to enumerate all partial states according to their length and dimension. The key idea is that we can "factor" a partial state uniquely as a concatenation of irreducible factors. Each new time that the partial state hits one of the borders of the tunnel, we start a new factor. For example, the factorization of the partial state of Figure 9 is shown in Figure 10

Definition 3.4 Let $P$ be the set of all partial states of robotic arms in a tunnel of width 2.

(a) A partial state of the robot is called irreducible if

- its first step is a horizontal link along the bottom border of the tunnel, and

- its final step is vertical or square, and is its first arrival to a border of the tunnel.

(b) A partial state of the robot is called irreducible final if it is empty or 

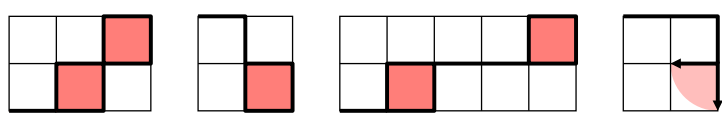

Fig. 10: The partial state of Figure 9 has a factorization of the form $M_{1} M_{5} M_{1} F_{10}$. (See Tables 1 and 2 )

- its first step is a horizontal link along the bottom border of the tunnel, and

- either it never arrives to a border, or it arrives in its final step, which is a claw.

Let $M$ and $F$ be the sets of irreducible and irreducible final partial states, respectively.

Let $\mathcal{S}=\bigcup_{n=0}^{\infty} \mathcal{S}_{n}$, and denote by $B^{*}$ the collection of all words that can be formed in an alphabet $B$. For instance, $a^{*}=\{\emptyset, a, a a, a a a, a a a a, \ldots\}$ and $\{a, b\}^{*}=\{\emptyset, a, b, a a, a b, b a, b b, a a a, a a b, \ldots\}$.

Proposition 3.5 The partial states in $\mathcal{S}$ starting with a right step $r$ are in weight-preserving bijection with the words in $M^{*} F$; that is, each partial state in $\mathcal{S}$ corresponds to a unique word of the form $m_{1} m_{2} \ldots m_{\ell} f$ with $m_{i} \in M$ and $f \in F$.

Proof: In view of Lemma 3.3, it is clear from the definitions that every partial state that starts with a horizontal step $r$ factors uniquely as a concatenation $m_{1}^{ \pm} m_{2}^{ \pm} \ldots m_{\ell}^{ \pm} f^{ \pm}$where each $m_{i} \in M, f \in F$, and $p^{ \pm}$equals $p$ or its reflection $p^{-}$across the horizontal axis. It remains to observe that whether $m_{i}^{ \pm}$is $m_{i}$ or $m_{i}^{-}$(and whether $p^{ \pm}$is either $p$ or $p^{-}$) is determined by the previous terms of the sequence.

Corollary 3.6 If the generating functions for partial states, irreducible partial states, and irreducible final partial states are $C(x, y), M(x, y), F(x, y)$ respectively, then

$$
1+x C(x, y)=\frac{F(x, y)}{1-M(x, y)}
$$

Proof: This follows from Proposition 3.5. The extra factor of $x$ comes from the fact that Proposition 3.5 is counting partial states with an initial right step.

\subsubsection{Enumeration of irreducible partial states.}

Proposition 3.7 The generating function for the irreducible partial states $M$ is

$$
M(x, y)=\frac{x^{3}+x^{4}+2 x^{4} y+2 x^{5} y+x^{5} y^{2}+x^{6} y^{2}}{(1-x)^{2}} .
$$

Proof: An irreducible partial state has exactly two symbols that contribute a vertical move, which can be either a $v$ or a $\square$. Thus there are 8 different families $M_{1}, \ldots, M_{8}$, corresponding to the irreducible partial states of the following form:

$$
\begin{aligned}
& \ldots \square \ldots \square \quad \ldots \square \ldots \square \square^{\prime} \quad \ldots \square \ldots v \quad \ldots \square \ldots v^{\prime} \\
& \ldots v \ldots \square \quad \ldots v \ldots \square^{\prime} \quad \ldots v \ldots v \quad \ldots v \ldots v^{\prime}
\end{aligned}
$$

where $\square^{\prime}$ and $v^{\prime}$ represent a move whose vertical step is in the opposite direction to the previous vertical step. Table 1 illustrates these 8 families together with their corresponding generating functions. 


\begin{tabular}{|l|l|l|}
\hline Type & Generating function \\
\hline$M_{1}=\left(r r^{*}\right) \square\left(r^{*}\right) \square$. & $\frac{x^{5} y^{2}}{(1-x)^{2}}$ \\
\hline$M_{2}=\left(r r^{*}\right) \square\left(r r^{*}\right) \square^{\prime}$. & $\frac{x^{6} y^{2}}{(1-x)^{2}}$ \\
\hline$M_{3}=\left(r r^{*}\right) \square\left(r^{*}\right) v$. & \\
\hline$M_{4}=\left(r r^{*}\right) \square\left(r r^{*}\right) v^{\prime}$ & \\
\hline$M_{5}=\left(r r^{*}\right) v\left(r^{*}\right) \square$. &
\end{tabular}

Tab. 1: Generating functions for the eight types of irreducible partial states.

Consider for example the family $M_{2}$. We must have at least one horizontal step before the first $\square$, and at least one horizontal step between the two $\square$ s, to make sure they do not intersect. Therefore the partial states in $M_{2}$ are given by $\left(r r^{*}\right) \square\left(r r^{*}\right) \square^{\prime}$, whose generating function is

$$
m_{2}(x, y)=\left(x \cdot \frac{1}{1-x}\right) x^{2} y\left(x \cdot \frac{1}{1-x}\right) x^{2} y=\frac{x^{6} y^{2}}{(1-x)^{2}} .
$$

The other formulas follow similarly. Thus $M(x, y)=m_{1}(x, y)+\cdots+m_{8}(x, y)$ is obtained by adding the generating functions in Table 1 .

\subsubsection{Enumeration of irreducible final partial states.}

Now let us compute the generating function $F(x, y)$ for irreducible final partial states.

Proposition 3.8 The generating function for the final irreducible partial states is

$$
F(x, y)=\frac{1+x^{2}+x^{4}+x^{2} y+2 x^{3} y+2 x^{4} y+x^{4} y^{2}+x^{5} y^{2}}{(1-x)^{2}}
$$

Proof: Each irreducible final partial state has at most one symbol among $\{v, \square\}$, and can possibly end with a symbol in $\{\llcorner, \ell\lrcorner$,$\} . Again, we let \left\llcorner^{\prime} \text { and }\right\lrcorner^{\prime}$ represent a move whose vertical step is in the opposite direction to the previous vertical step. Table 2 shows the 10 different families of possibilities together with their corresponding generating functions.

The generating function of $F$ is $f(x, y)=f_{1}(x, y)+\cdots+f_{10}(x, y)$. The result is then obtained by adding the generating functions in Table 2 . 
The configuration space of a robotic arm in a tunnel of width 2

\begin{tabular}{|l|l|l|}
\hline Irreducible move & Illustration & Generating function \\
\hline$F_{1}=r^{*}$ & & $\frac{1}{1-x}$ \\
\hline$F_{2}=\left(r r^{*}\right) \square\left(r^{*}\right)$ & \\
\hline$F_{3}=\left(r r^{*}\right) \square\left(r^{*}\right)\llcorner$ & \\
\hline$F_{4}=\left(r r^{*}\right) \square\left(r r^{*}\right)\left\llcorner^{\prime}\right.$ & \\
\hline$F_{5}=\left(r r^{*}\right) v\left(r^{*}\right)$ & \\
\hline$F_{6}=\left(r r^{*}\right) v\left(r^{*}\right)\llcorner$ & \\
\hline$F_{7}=\left(r r^{*}\right) v\left(r r^{*}\right)\left\llcorner^{\prime}\right.$ & \\
\hline$F_{8}=\left(r r^{*}\right)\llcorner$ & \\
\hline$F_{9}=\left(r^{2} r^{*}\right) v \ell$ & \\
\hline$\left.F_{10}=\left(r^{2} r^{*}\right) v\right\lrcorner$ & \\
\hline
\end{tabular}

Tab. 2: Generating functions for the ten types of irreducible final partial states..

Theorem 3.9 Let $\mathcal{S}_{n}$ be the configuration space for the robot of length $n$ moving in a rectangular tunnel of width 2. If $c_{n, d}$ denotes the number of d-dimensional cubes in $\mathcal{S}_{n}$, then

$$
C(x, y)=\sum_{n, d \geq 0} c_{n, d} x^{n} y^{d}=\frac{1+x^{2}+2 x^{3}-x^{4}+x y+x^{2} y+4 x^{3} y+x^{4} y+x^{3} y^{2}+2 x^{4} y^{2}+x^{5} y^{2}}{1-2 x+x^{2}-x^{3}-x^{4}-2 x^{4} y-2 x^{5} y-x^{5} y^{2}-x^{6} y^{2}}
$$

Proof: This follows from Corollary 3.6 and Propositions 3.7 and 3.8

Theorem 3.10 The Euler characteristic of the configuration space $\mathcal{S}_{n}$ equals 1.

Proof: Since the Euler characteristic of $\mathcal{S}_{n}$ is $\chi\left(\mathcal{S}_{n}\right)=\sum_{d \geq 0}(-1)^{d} c_{n, d}$, the generating function for $\chi\left(\mathcal{S}_{n}\right)$ is given by substituting $y=-1$ into Theorem 3.9 .

$$
\begin{aligned}
\sum_{n \geq 0} \chi\left(\mathcal{S}_{n}\right) x^{n} & =\sum_{n \geq 0}\left(\sum_{d \geq 0}(-1)^{d} c_{n, d}\right) x^{n}=C(x,-1) \\
& =\frac{1-x-x^{3}+x^{5}}{1-2 x+x^{2}-x^{3}+x^{4}+x^{5}-x^{6}} \\
& =\frac{1}{1-x}=1+x+x^{2}+x^{3}+\ldots,
\end{aligned}
$$

in an expected but still beautiful miracle of cancellation. All the coefficients of this series are equal to 1, as desired. 


\section{Posets with inconsistent pairs (PIPs)}

Determining whether a metric space $X$ is $\operatorname{CAT}(0)$ is a rather subtle question in general; it should be clear that Definition 2.2 is not a very tractable method, as it would require checking the length of every chord in every triangle in $X$. Fortunately, Gromov [7] showed that when $X$ is a cubical complex, this metric property has the combinatorial and topological characterization stated in Theorem 3.1 . In fact, for finite $\mathrm{CAT}(0)$ cubical complexes, there are several combinatorial characterizations [4, 10, 11, 12]. We will use the one given by Ardila, Owen, and Sullivant in [4], which we now describe.
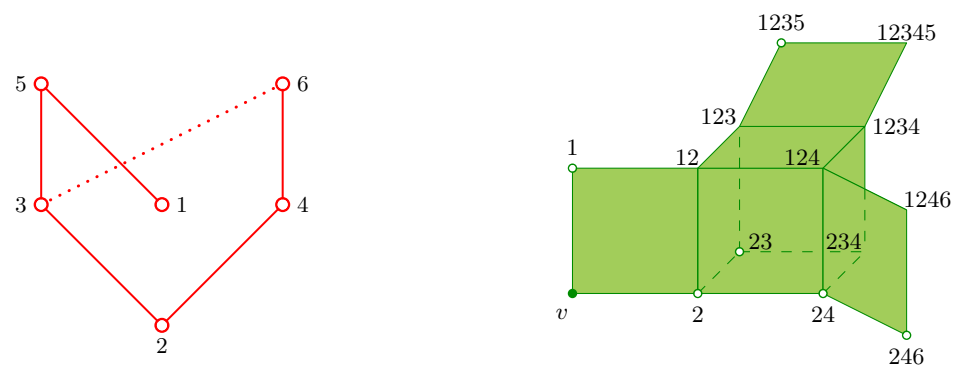

Fig. 11: A PIP and the corresponding rooted CAT(0) cubical complex.

Definition 4.1 A poset with inconsistent pairs (PIP) is a locally finite poset $P$ of finite width, together with a collection of inconsistent pairs, which we denote $p \leftrightarrow q$ (where $p \neq q)$, such that

$$
\text { if } p \leftrightarrow q \text { and } q<q^{\prime} \text { then } p \leftrightarrow q^{\prime} \text {. }
$$

The Hasse diagram of a poset with inconsistent pairs (PIP) is obtained by drawing the poset, and connecting each <-minimal inconsistent pair with a dotted line. The left panel of Figure 11 shows an example.

Recall that an order ideal of $P$ is a subset $I$ such that if $x<y$ and $y \in I$ then $x \in I$. We say that $I$ is consistent if it contains no inconsistent pair.

Each PIP $P$ gives rise to a rooted cube complex, which we denote $X(P)$. The vertices of $X(P)$ are identified with the consistent order ideals of $P$. There will be a cube $C(I, M)$ for each pair $(I, M)$ of a consistent order ideal $I$ and a subset $M \subseteq I_{\max }$, where $I_{\max }$ is the set of maximal elements of $I$. This cube has dimension $|M|$, and its vertices are obtained by removing from $I$ the $2^{|M|}$ possible subsets of $M$. The cubes are naturally glued along their faces according to their labels. The root is the vertex corresponding to the empty order ideal. Figure 11 shows a PIP $P$ and the corresponding complex $X(P)$, which is rooted at $v$. For example, the compatible order ideal $I=\{1,2,3,4\}$ and the subset $M=\{1,4\} \subseteq I_{\max }$ gives rise to the square with vertices labelled 1234, 123, 234, 23 .

Theorem 4.2 [4] The map $P \mapsto X(P)$ is a bijection between posets with inconsistent pairs and rooted $\mathrm{CAT}(0)$ cube complexes.

Theorem 4.2 offers a useful technique to prove that a configuration space is a $\operatorname{CAT}(0)$ cubical complex; we "simply" have to choose a root for it, and find the corresponding PIP. Of course, carrying this task out is a non-trivial matter, as will soon become apparent. We propose the following solution for the robotic arm in a tunnel of width 2 . 
Definition 4.3 Define the coral PIP $C_{n}$ as follows.

- The elements are non-decreasing $k$-tuples $\left(a_{1}, \ldots, a_{k-1} ; a_{k}\right)$ such that $a_{k} \leq n$ and

$$
a_{i+1}-a_{i} \equiv \begin{cases}1 \bmod 4 & \text { for } i=1 \\ 3 \bmod 4 & \text { for } 2 \leq i \leq k-2 \\ 0 \operatorname{or} 2 \bmod 4 & \text { for } i=k-1\end{cases}
$$

- The element $\left(a_{1}, \ldots, a_{k-1} ; a_{k}\right)$ is covered by at most 3 elements:

$$
\begin{array}{rll}
\left(a_{1}-1, \ldots, a_{k-1}-1 ; a_{k}-1\right) & \text { if } & a_{1} \geq 2 \\
\left(a_{1}, \ldots, a_{k-1} ; a_{k}+2\right) & \text { if } & a_{k} \leq n-2 \\
\left(a_{1}, \ldots, a_{k-1}, a_{k}+1 ; a_{k}+1\right) & \text { if } & a_{k}-a_{k-1} \equiv 2 \bmod 4
\end{array}
$$

- The minimal inconsistent pairs are

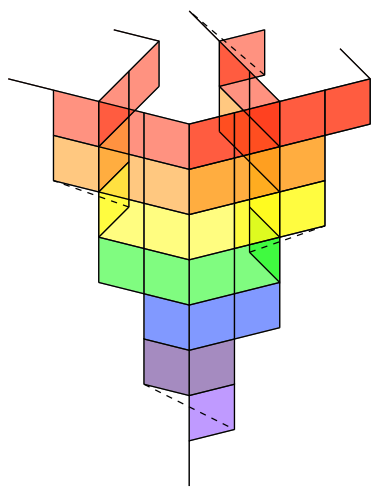

$$
\left(a_{1}, \ldots, a_{k-1} ; n\right) \leftrightarrow\left(a_{1}+1, \ldots, a_{k-1}+1, n ; n\right)
$$

The colored figure above illustrates the coral PIPs for $n=1,2, \ldots, 9$; every time we add a new link to the arm, we add a new layer (indicated by a new color) to the PIP.

The element $\left(a_{1}, \ldots, a_{k-1} ; a_{k}\right)$ of the PIP corresponds to the position of the arm which has vertical steps $a_{1}, a_{1}+2, a_{1}+4, \ldots, a_{2}-1$ (in row 1), $a_{2}, a_{2}+2, a_{2}+4, \ldots, a_{3}-1$ (in row 2), $a_{3}, a_{3}+2, a_{3}+$ $4, \ldots, a_{4}-1$ (in row 1$), \ldots, a_{k-1}, a_{k-1}+2, a_{k-1}+4, \ldots, a_{k}$ (in row $\left.(k-1 \bmod 2)\right)$.

Definition 4.4 The extended coral PIP $\bar{C}_{n}$ is obtained from the coral PIP $C_{n}$ by adding two states $L_{1}=$ $(n-1 ; \underline{n})$ and $L_{2}=(n-5, n-4 ; \underline{n})$, where

- $L_{1}$ covers $(n-1, n ; n)$ and is inconsistent with $(n-2 ; n)$, and

- $L_{2}$ covers $(n-4, n-3, n ; n)$ and $(n-5, n-4 ; n-4)$ and is inconsistent with $(n-5, n-4 ; n)$.

The vertices $L_{1}$ and $L_{2}$ correspond to the two simplest positions of the arm having a left step, namely, $r \ldots r u l$ and $r$...ruurrdl respectively.

Theorem 4.5 The configuration space $\mathcal{S}_{n}$ of the robotic arm of length $n$ in a tunnel of width 2 is a CAT(0) cubical complex. Its corresponding PIP is the extended coral PIP $\bar{C}_{n}$ of Definition 4.4

One can verify directly that the bijection $P \mapsto X(P)$ of Theorem 4.2 sends the coral PIP $C_{n}$ to the configuration space $\mathcal{S}_{n}$. However, the proof becomes simpler when it is framed in a more general setting.

In an upcoming paper [3] we study the robotic arm of length $n$ with no left steps in a tunnel of any width, and prove that its configuration space is a CAT $(0)$ cubical complex. The more general coral PIP, which generalizes Definition 4.3, is described in terms of combinatorial objects called coral snake tableaux. For details and proofs, see [3].

\section{Implementation of the shortest path algorithm}

Theorem 5.1 [1] 2] 9] If the configuration space of a robot is a CAT(0) cubical complex, there is an algorithm to move the robot optimally from one position to another, in terms of:

- the number of moves, if only one move at a time is allowed,

- the number of steps (where in each step we may perform several physically independent moves),

- time elapsed. 
These algorithms are described in detail in [2]; we have implemented them for the robotic arms discussed in this paper. More details may be found at:

http://math.sfsu.edu/federico/Articles/movingrobots.html

\section{Acknowledgements}

This paper includes results from HB's undergraduate thesis at Universidad del Valle in Cali, Colombia (advised by FA and CC) and JG's undergraduate project at San Francisco State University in San Francisco, California (advised by FA). We thank the SFSU-Colombia Combinatorics Initiative, which made this collaboration possible. FA would also like to thank the Pacific Ocean for bringing him the coral that inspired Theorem 1.5

\section{References}

[1] A. Abrams and R. Ghrist. State complexes for metamorphic robots. Int. J. Robotics Res. 23 (2004) 811-826.

[2] F. Ardila, T. Baker, and R. Yatchak. Moving robots efficiently using the combinatorics of CAT $(0)$ cubical complexes. Adv. in Appl. Math. 48 (2012) 142-163.

[3] F. Ardila, H. Bastidas, C. Ceballos, J. Guo. The configuration space of a robotic arm in a tunnel. In preparation, 2016.

[4] F. Ardila, M. Owen, and S. Sullivant. Geodesics in CAT(0) cubical complexes. SIAM J. Discrete Math. 28-2 (2014) 986-1007.

[5] M. Bridson and A. Haefligher. Metric spaces of non-positive curvature. Springer-Verlag, Berlin, 1999.

[6] R. Ghrist and V. Peterson. The geometry and topology of reconfiguration. Adv. in Appl. Math. 38 (2007) 302-323.

[7] M. Gromov. Hyperbolic groups. In Essays in group theory, volume 8 of Math. Sci. Res. Inst. Publ. 75- 263. Springer, New York, 1987.

[8] G.A. Niblo and L.D. Reeves. The geometry of cube complexes and the complexity of their fundamental groups. Topology, 37(3) (1998) 621-633.

[9] L. D. Reeves. Biautomatic structures and combinatorics for cube complexes. Ph.D. thesis, University of Melbourne, 1995.

[10] M. A. Roller. Poc sets, median algebras and group actions. an extended study of Dunwoody's construction and Sageev's theorem. Unpublished preprint, 1998.

[11] M. Sageev. Ends of group pairs and non-positively curved cube complexes. Proc. London Math. Soc. (3), 71 (3) 585617, 1995.

[12] L. Santocanale. A nice labelling for tree-like event structures of degree 3. Inf. Comput., 208:652-665, 2010. 